\title{
Diagnostic value of high-frequency ultrasound and magnetic resonance imaging in early rheumatoid arthritis
}

\author{
MING-YU WANG ${ }^{1}$, XIAN-BIN WANG ${ }^{1}$, XUE-HUI SUN $^{1}$, FENG-LI LIU $^{2}$ and SHENG-CHUAN HUANG ${ }^{3}$ \\ Departments of ${ }^{1}$ Rheumatology and Immunology, ${ }^{2}$ Rheumatology and Radiology and ${ }^{3}$ Rheumatology and Ultrasonography, \\ Yantai Yuhuangding Hospital, Yantai, Shandong 264000, P.R. China
}

Received April 21, 2015; Accepted May 19, 2016

DOI: $10.3892 /$ etm.2016.3695

\begin{abstract}
Early diagnosis and management improve the outcome of patients with rheumatoid arthritis (RA). The present study explored the application of high-frequency ultrasound (US) and magnetic resonance imaging (MRI) in the detection of early RA. Thirty-nine patients (20 males and 19 females) diagnosed with early RA were enrolled in the study. A total of 1,248 positions, including 858 hand joints and 390 tendons, were examined by high-frequency US and MRI to evaluate the presence of bone erosion, bone marrow edema (BME), synovial proliferation, joint effusion, tendinitis and tendon sheath edema. The imaging results of the above abnormalities, detected by US, were compared with those identified using MRI. No statistically significant overall changes were observed between high-frequency US and MRI in detecting bone erosion [44 (5.1\%) vs. 35 (4.1\%), respectively; $\mathrm{P}>0.05]$, tendinitis [18 (4.6\%) vs. 14 $(1.5 \%)$, respectively; $\mathrm{P}>0.05]$ and tendon sheath edema [37 $(9.5 \%)$ vs. 30 (7.7\%), respectively; P>0.05]. Significant differences were observed between high-frequency US and MRI with regards to the detection of synovial proliferation [132 $(15.4 \%)$ vs. $66(7.7 \%)$, respectively; $\mathrm{P}<0.05]$ and joint effusion [89 (10.4\%) vs. 52 (6.1\%), respectively; $\mathrm{P}<0.05]$. In addition, significant differences were identified between the detection of BME using MRI compared with high-frequency US (5.5 vs. 0\%, respectively; $\mathrm{P}<0.05$ ). MRI and high-frequency US of the dominant hand and wrist joints were comparably sensitive to bone erosion, tendinitis and tendon sheath edema. However, MRI was more sensitive in detecting bone marrow edema in early RA, while US was more sensitive in the evaluation of joint effusion and synovial proliferation. In conclusion, US and MRI are promising for the detection and diagnosis of inflammatory activity in patients with RA.
\end{abstract}

Correspondence to: Professor Xian-Bin Wang, Department of Rheumatology and Immunology, Yantai Yuhuangding Hospital, 20 Yuhuangding East Road, Yantai, Shandong 264000, P.R. China E-mail: wangxianbin02@163.com

Key words: rheumatoid arthritis, high frequency ultrasonography, magnetic resonance imaging, bone erosion, synovial proliferation

\section{Introduction}

Rheumatoid arthritis (RA) is a chronic and common inflammatory disease affecting as much as $1 \%$ of the population worldwide (1). It is a destructive and progressive joint disease characterized by synovial membrane inflammation and joint cartilage destruction, which results in joint deformity and disability, and an impaired quality of life (2). The occurrence of RA is more common in females compared with males (1). Previous studies have indicated that patients with RA are likely to experience irreversible joint damage 1 year after its onset (3). Thus, the early diagnosis and treatment of RA are important for improved outcomes of RA.

Magnetic resonance imaging (MRI) and ultrasound (US) techniques, which provide the satisfactory visualization of joint structures coupled with high sensitivity and specificity, are commonly used in the clinical examination and radiographic assessment of RA (4). MRI allows direct visualization of synovitis, together with prognostic information of neighboring bones and bone marrow, which provides crucial evidence of bone erosion and bone marrow edema (5). Accumulating evidence has indicated that MRI is more sensitive than conventional radiography in identifying bone erosion and edema (6,7). Døhn et al (8) indicated that MRI was $\sim 5$ times more sensitive than X-ray in the detection of bone erosion by comparing MRI and X-ray of the second-to-fifth metacarpophalangeal (MCP) joints of one hand in 17 patients with RA. In addition, a previous report demonstrated that MRI scan images could predict the onset of RA with high sensitivity (100\%) and specificity (78\%) in $31 / 33$ patients, and evaluate the synovitis with erosion and/or bone edema in a previous similar group of patients with RA (9).

Although US can not detect bone marrow edema, it is used frequently for the clinical assessment of synovitis with effusion, power Doppler signal and synovial hypertrophy (10). In a previous study, Zheng et al (11) confirmed that US examination was feasible and capable of predicting RA disease, as measured by the disease activity score in 28 joints for the evaluation of joints. Furthermore, McQueen et al (12) have previously indicated that US is able to detect bone erosion with $95 \%$ specificity and $78 \%$ predictive accuracy, with a bone volume loss of $>20 \%$ in US-accessible areas, such as in the dorsal and palmar aspects of MCP joints. These results 
indicate that MRI and US may be applicable in the evaluation of RA activity, progression and treatment responses.

In the present study, the effectiveness of high-frequency US is compared with MRI in the detection of early RA, and the advantages of each method, with regards to joint effusion, synovial proliferation and bone marrow edema, are identified.

\section{Materials and methods}

Patients. A total of 39 patients (20 men and 19 women; mean age, 50.2 years) diagnosed with early-stage RA between January and December 2010 in Yantai Yuhuangding Hospital (Yantai, China) were enrolled in the current study. The diagnosis of RA was based on the 1987 American College of Rheumatology classification criteria (13). Patients who had experienced symptoms of RA for $>24$ months were excluded from the study. The median age of patients at the onset of the disease was $51.8 \pm 2.2$ years (range, 22-75 years). The mean disease course was $8.8 \pm 2.5$ months. Written informed consent was obtained from all the participants. The study was conducted with approval from the Ethics Committee of Yantai Yuhuangding Hospital.

US examination. Ultrasonographic evaluation was performed using color US diagnostic apparatus (LOGIQ-10; General Electric Company, Fairfield, CT, USA), equipped with a $12-\mathrm{MHz}$ linear array probe. US was conducted by an experienced radiologist blinded to the study. The patients were examined while sitting upright, with the hand placed on a cushion and the palm facing upward. A total of 1,248 joints were scanned, including bilateral wrist joints, and the sagittal and coronal sections of the hand joints. All patients underwent US assessment of the wrists, MCP joints, proximal interphalangeal (PIP) joints for the presence of joint effusion (defined by the compressible anechoic intracapsular region), bone erosion (defined by bone cortex breakage in the joint area) and synovial proliferation (defined by thickness $>2 \mathrm{~mm}$ in wrist joints, and $>1 \mathrm{~mm}$ in MIP and PIP joints with a low echo) $(14,15)$. Lateral flexion of MCP joints 2-5 and wrists were examined for the presence of tendinitis and tendon sheath edema. In addition, synovial blood flow in each joint was assessed with color Doppler flow imaging and high-resolution color flow.

MRI evaluation. All patients underwent MRI evaluation of hands and wrists using a 3.0T MR system (Signa EXCITE; General Electric Company). Patients were placed in a prone position with the hand above the head and the palm facing upward. Continuous coronal and axial plane MRI without contrast were obtained using T1-weighted spin-echo sequences, short inversion time inversion recovery (STIR) gradient-echo sequences and T2-weighted turbo spin-echo sequences, respectively. The presence of erosion, bone marrow edema, synovial thickening and tendinitis were assessed according to the Outcome Measures in Rheumatology RA MRI Scoring system (5). MRI images were evaluated by a doctor who was experienced in joint MRI.

Statistical analysis. Data analysis was performed using SPSS version 16.0 software (SPSS, Inc., Chicago, IL, USA). Data are expressed as the mean \pm standard deviation and were analyzed using $\chi^{2}$ test. $\mathrm{P}<0.05$ was considered to indicate a statistically significant difference.

\section{Results}

High-frequency US is significantly better at detecting synovial proliferation and joint effusion, compared with MRI. US and MRI results obtained from 39 patients are presented in Table I. A total of 858 joints were scanned by US and MRI for the detection of bone erosion, bone marrow edema, synovial proliferation and joint effusion, while the other 390 tendons were used for the evaluation of tendinitis and tendon sheath edema. No statistically significant sensitivities were observed between high-frequency US and MRI in bone erosion [44 (5.1\%) vs. 35 (4.1\%), respectively; $\mathrm{P}>0.05$ ], tendinitis [18 (4.6\%) vs. $14(1.5 \%)$, respectively; $\mathrm{P}>0.05]$ and tendon sheath edema [37 $(9.5 \%)$ vs. 30 (7.7\%), respectively; $\mathrm{P}>0.05]$. Significant differences were identified between high-frequency US and MRI in synovial proliferation [132 $(15.4 \%)$ vs. $66(7.7 \%)$, respectively; $\mathrm{P}<0.05]$ and joint effusion [89 (10.4\%) vs. $52(6.1 \%)$, respectively; $\mathrm{P}<0.05$ ]. Significantly higher sensitivity $(\mathrm{P}<0.05)$ was identified in the detection of bone marrow edema using MRI (5.5\%) compared with high-frequency US (0\%).

MRI is suitable for visualization of bone marrow edema in early RA, and US is sensitive to the detection of joint effusion and synovial proliferation. The symptoms of the above six abnormalities in RA are presented in Fig. 1. The images provide an insight into which types of imaging can provide the required information in specific practice situations. Prominent erosion in the distal third metacarpal bone with discontinuous bone density (red arrow) was identified by US (Fig. 1A), while it was presented as moderate hypointensity on a T1-weighted image (T1WI, red arrow) and hyperintensity on a T2-weighted image (T2WI, red arrow) in the coronal plane of the distal third metacarpal bone on MRI images (Fig. 1B and C). Increased width in the tendon (green arrow) with disturbance echo and a thin sheath layer low echo (red arrow) were identified in the flexor tendon of the left hand, which indicates tendinitis and tendon sheath edema using high-frequency US (Fig. 1D). Meanwhile, dilated tendon and high intensity on T2WI (red arrow) in the surroundings of the tendon were observed in the sagittal planes of the left-hand flexor tendon on the MRI images (Fig. 1E). Joint effusion with low echo, synovial proliferation in a cotton-like shape and blood flow signal in the synovium (red arrow) were identified in the bone gap of the right wrist with US (Fig. 1F). Joint effusion and synovial proliferation were presented as hyperintensity on T2WI MRI images (red arrow) in the coronal position of the bone gap of the right wrist (Fig. 1G). Bone marrow edema appeared as a hyposignal on the T1-weighted sequence image, and hypersignal on T2-weighted sequence image (red arrow), in the coronary carpal bone of the left hand (Fig. 1H and I), whereas bone marrow abnormality was not visualized using US. These results suggest that MRI is ideally suited to visualizing bone marrow edema in early RA, whereas US has high sensitivity and diagnostic values in the detection of joint effusion and synovial proliferation. 
Table I. Comparisons between high-frequency US- and MRI-detected abnormalities in joints ( $\mathrm{n}=858)$ in rheumatoid arthritis.

\begin{tabular}{lccrr}
\hline Item & No. joints & US (\% total joints) & MRI (\% total joints) & P-value \\
\hline Bone erosion & 858 & $44(5.1 \%)$ & $35(4.1 \%)$ & 0.300 \\
Bone marrow edema & 858 & $0(0.0 \%)$ & $47(5.5 \%)$ & $<075$ \\
Synovial proliferation & 858 & $132(15.4 \%)$ & $66(7.7 \%)$ & 48.324 \\
Joint effusion & 858 & $89(10.4 \%)$ & $52(6.1 \%)$ & $<0.001$ \\
Tendinitis & 390 & $18(4.6 \%)$ & $14(1.5 \%)$ & 0.001 \\
Tendon sheath edema & 390 & $37(9.5 \%)$ & $30(7.7 \%)$ & 0.470 \\
\end{tabular}

US, ultrasound; MRI, magnetic resonance imaging.

A

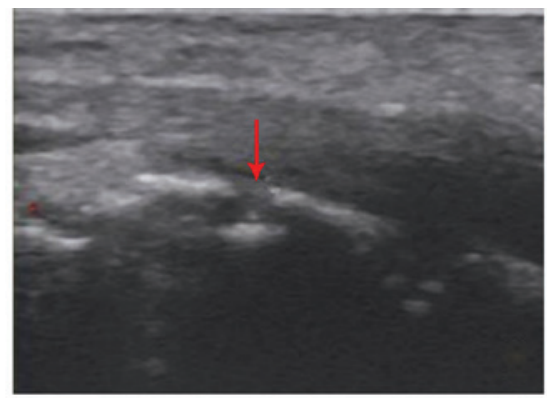

D



G

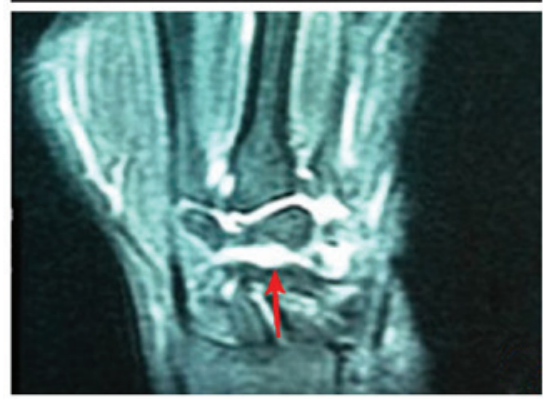

B
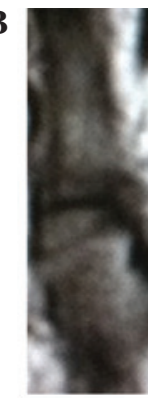

E



\section{$\mathbf{H}$}



C
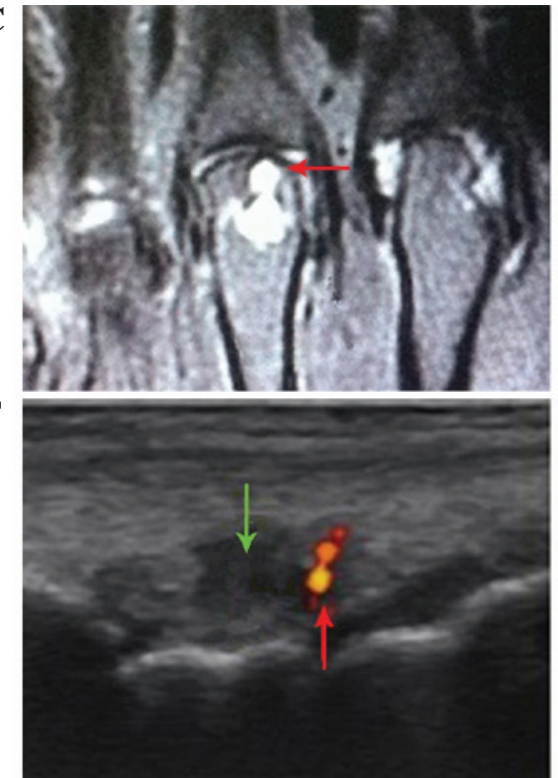

I

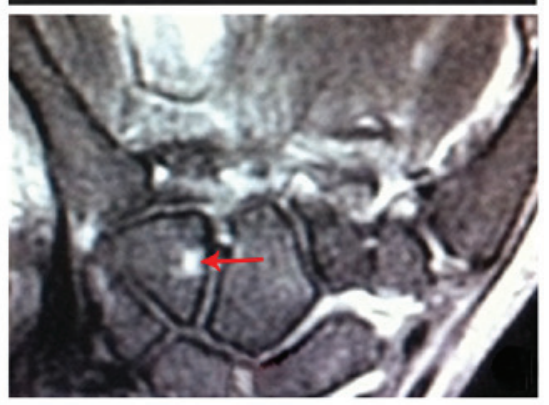

Figure 1. Symptoms of abnormalities in rheumatoid arthritis with magnetic resonance imaging and high-frequency ultrasound. (A) Bone erosion (red arrow) indicated by ultrasound examination. (B) Bone erosion (red arrow) indicated by MRI featured by moderate signal intensities in the T1-weighted image. (C) Bone erosion (red arrow) indicated by MRI featured moderate signal intensities in the T2-weighted image. (D) Presence of tendonitis on the left hand by ultrasound examination, which demonstrated widening of the tendon (green arrow) and low intensity echoes around the tendon (red arrow). (E) Presence of tendonitis on the left hand indicated by MRI, which showed widening of tendon and high intensity T2-weighted image signals around the tendon (red arrow). (F) Presence of articular dropsy in the right wrist by ultrasound examination (red arrow). (G) Dropsy in the intercarpal joints and synovial proliferation indicated by MRI. (H) Presence of bone marrow edema by MRI, featured by low intensity T1-weighted image signals (red arrow). (I) Presence of bone marrow edema by MRI, featured by low intensity T2-weighted image signals (red arrow). MRI, magnetic resonance imaging.

\section{Discussion}

RA is a form of autoimmune arthritis manifested with persistent synovitis that most commonly involves the wrist and finger joints (2). RA occurs across all ethnic populations and ages, with a peak incidence between the ages of 45 and 65 years (16). Uncontrolled RA may result in permanent joint injury and other extra-articular complications affecting normal activities and quality of life (17). Aside from genetic factors, environmental factors are prominent in the development of RA (18). For example, smoking is associated with a substantially increased risk of RA (19), and patients experiencing long-term stress are prone to developing RA (20). The early and accurate diagnosis of RA serves an important 
role in the assessment of disease progression and the early introduction of effective treatment options (21). Accumulating evidence indicates that the early diagnosis of RA contributes towards the improved outcome of RA, particularly within the first three months of the onset of RA (22). Therefore, the identification of changes associated with early RA using imaging techniques is important in clinical practice.

To date, imaging techniques have served a focal role in the evaluation of disease progression and the response to therapy in RA (4). Plain radiography has been traditionally used to detect and quantify RA, including the detection of joint space loss, soft-tissue swelling and marginal erosion (23). However, the obtained information from X-ray analysis relies on relatively late disease features, and is unsatisfactory with regards to current demands, validity and accuracy.

Currently, US and MRI are commonly used in the diagnosis and management of rheumatic disease (4). MRI provides a more global view of the articular surfaces and internal bone structure of joints compared with US. MRI enables the direct visualization of numerous bone and soft tissue changes involving the synovial membrane, bone erosion and cartilage thinning (24). Additionally, MRI has a unique capacity to detect inflammation within bone in the form of bone marrow edema, and this provides important diagnostic and prognostic information in patients with RA. US is widely used in the early diagnosis of RA, particularly in the introduction and application of high frequency color Doppler US, which can monitor joint effusion, blood flow change in the synovium and synovial thickening (25). Furthermore, US may be used to monitor disease status and predict disease recurrence, providing an effective and valuable evaluation of treatments (26). Furthermore, US is more acceptable for patients, as it is relatively simple, cheap and does not use radiation (4). The combination of US and MRI for the diagnosis of early RA has been validated (27). Szkudlarek et al (28) reported that the sensitivity and specificity of Doppler US were 88.8 and $97.9 \%$, respectively, which is as effective as dynamic MRI for the evaluation of synovitis in MCP joints. Rahmani et al (29) demonstrated that the overall sensitivity and specificity of US in detecting bone erosion were 63 and 98\%, respectively. Importantly, MRI and US have been described as indispensable diagnostic tools to evaluate disease progression and the response of disease, including $\mathrm{RA}$, to various types of treatments.

The decision of which modality should be used in clinical diagnosis should be taken into consideration due to the coexistence of the strengths and weaknesses of US and MRI. It is important to understand the specific performance of US and MRI with regards to imaging joint abnormalities. Bone marrow edema is understood to be a marker for early inflammatory changes resulting from cellular infiltration into bone marrow (30). The visualization of bone marrow edema (BME) is clinically important in RA as it is the strongest predictive factor in the progression of bone erosion (31). BME on MRI images is identified as a lesion with intact trabecular structures and ill-defined margins (32). It presents high signal intensity on STIR or T2-weighted sequences, and presents low signal intensity on T1-weighted MRI images (5). The ability of MRI to detect BME is important since the pathologies of BME can not be visualized by US.
Bone erosion, a central feature and common finding in RA, is associated with disease severity and poor functional outcome (33). The presence of erosion detected by MRI is identified by direct contact with cortical bone with sharp margins and obvious destruction of the cortical bone barrier (34). Erosion presents as hyperintensity on STIR or T2-weighted sequences and hypointensity on T1-weighted sequences (34). In addition, it is described as an intra-articular discontinuity of the bone surface that is visible in two perpendicular planes on US images (15). It is controversial with regards to which image modality has superior sensitivity in the detection of bone erosion. Magnani et al (35) suggested that US was at least as sensitive as MRI in detecting bone erosion in wrist and MCP joints, while other evidence indicated that MRI had significantly increased sensitivity compared with US for the evaluation of erosion in the wrist and hand (36). Consistent with the previous report (34), the present study observed no significant difference between high-frequency US and MRI with regards to the detection of bone erosion. Soft tissues are often present in joint surroundings, such as in the tendon and tendon sheath, resulting in tendinitis and tendon sheath edema, which are common complications of RA that typically occur in the hands and wrists (37). The abnormalities typically present as dilated tendons with irregular margins, accompanied with a disturbed fibrous-like echo (38). In the present study, although high-frequency US demonstrated slightly increased sensitivities with regards to detecting tendinitis and tendon sheath edema, no statistically significant differences were identified compared with MRI results.

Proliferative synovitis is the earliest and most important pathological change observed in RA, and it is associated with the quantity of joint fluid present (39). The presence of proliferative synovitis on MRI is identified by a thickened area of synovial compartment that presents as hypointensity on T1-weighted sequences and hyperintensity on T2-weighted sequences (40). Although it is often less bright on fluid-sensitive sequences in comparison with joint fluid, it can not be easily differentiated from joint fluid based on the presence of similar weighted sequences. Proliferative synovitis detected by US presents as abnormal hypoechoic intra-articular tissue that is poorly compressible and non-displaceable with Doppler signal exhibition (15). The non-displaceable nature of proliferative synovitis enables US to distinguish between joint fluid and synovial thickening. Kasukawa et al (41) indicated that US was easy to use and effective in estimating the degree and pattern of synovial proliferation and synovial effusion in the knee joints of patients with RA. In the current study, it was demonstrated that US was significantly more sensitive in detecting synovial proliferation and joint effusion compared with MRI.

Providing effective treatments for RA following the onset of the disease is essential in order to avoid the risk of long-term structural and functional damage. To date, therapies used to treat RA include non-steroidal anti-inflammatory drugs, which are used for the treatment of pain and inflammation, and disease-modifying antirheumatic drugs, which are commonly used as first-line therapy for all newly diagnosed cases of RA (42). In addition, glucocorticoids and other biological agents are used as a treatment strategy for RA $(43,44)$. Furthermore, non-pharmacological and non-surgical interventions are increasingly optimized in patients with RA, including 
comprehensive occupational therapy, wrist working splints and finger splints (45).

In conclusion, the results in the present study indicated that high-frequency US and MRI are both effective in detecting bone erosion, tendinitis and tendon sheath edema in patients with early RA. MRI was demonstrated to be superior in the evaluation of bone marrow edema, while high-frequency US demonstrated an increased sensitivity for detecting early joint effusion and synovial proliferation in comparison with MRI. As further evidence becomes available, US and MRI will become increasingly important in the diagnosis and management of early RA. High-frequency US may be considered as a valuable modality for the detection of early RA, particularly when MRI is not accessible. The decision of which tool should be used in a given trial should rely on the clinical output requirement in order to minimize patient discomfort.

\section{References}

1. Gibofsky A: Overview of epidemiology, pathophysiology and diagnosis of rheumatoid arthritis. Am J Manag Care 18 (Suppl 13): S295-S302, 2012

2. Apfelberg D, Maser R, Lash H, Kaye R, Britton M and Bobrove A: Rheumatoid hand deformities: Pathophysiology and treatment. West J Med 129: 267-272, 1978.

3. Machold KP, Stamm TA, Nell VP, Pflugbeil S, Aletaha D, Steiner G, Uffmann M and Smolen JS: Very recent onset rheumatoid arthritis: Clinical and serological patient characteristics associated with radiographic progression over the first years of disease. Rheumatology (Oxford) 46: 342-349, 2007.

4. Khan KM, Forster BB, Robinson J, Cheong Y, Louis L, Maclean L and Taunton JE: Are ultrasound and magnetic resonance imaging of value in assessment of Achilles tendon disorders? A two year prospective study. Br J Sports Med 37: 149-153, 2003.

5. Østergaard M, Peterfy C, Conaghan P, McQueen F, Bird P,Ejbjerg B, Shnier R, O'Connor P, Klarlund M, Emery P, et al: OMERACT rheumatoid arthritis magnetic resonance imaging studies. Core set of MRI acquisitions, joint pathology definitions and the OMERACT RA-MRI scoring system. J Rheumatol 30: 1385-1386, 2003.

6. Ostendorf B, Scherer A, Mödder U and Schneider M: Diagnostic value of magnetic resonance imaging of the forefeet in early rheumatoid arthritis when findings on imaging of the metacarpophalangeal joints of the hands remain normal. Arthritis Rheum 50: 2094-2102, 2004.

7. Suter L, Fraenkel L and Braithwaite R: The role of magnetic resonance imaging in the diagnosis and prognosis of rheumatoid arthritis. Arthritis Care Res (Hoboken) 63: 675-688, 2011.

8. Døhn UM, Ejbjerg BJ, Hasselquist M, Narvestad E, Court-Payen M, Szkudlarek M, Møller J, Thomsen HS and Ostergaard M: Rheumatoid arthritis bone erosion volumes on CT and MRI Reliability and correlations with erosion scores on CT, MRI and radiography. Ann Rheum Dis 66: 1388-1392, 2007.

9. Narváez J, Sirvent E, Narváez JA, Bas J, Gómez-Vaquero C, Reina D, Nolla JM and Valverde J: Usefulness of magnetic resonance imaging of the hand versus anticyclic citrullinated peptide antibody testing to confirm the diagnosis of clinically suspected early rheumatoid arthritis in the absence of rheumatoid factor and radiographic erosion. Semin Arthritis Rheum 38: 101-109, 2008.

10. Backhaus M, Burmester G, Sandrock D, Loreck D, Hess D, Scholz A, Blind S, Hamm B and Bollow M: Prospective two year follow up study comparing novel and conventional imaging procedures in patients with arthritic finger joints. Ann Rheum Dis 61: 895-904, 2002.

11. Zheng G, Wang L, Jia X, Li F, Yan Y, Yu Z, Li L, Wei Q and Zhang F: Application of high frequency color doppler ultrasound in the monitoring of rheumatoid arthritis treatment. Exp Ther Med 8: 1807-1812, 2014.

12. McQueen FM: Imaging in early rheumatoid arthritis. Best Pract Res Clin Rheumatol 27: 499-522, 2013.

13. Arnett FC, Edworthy SM, Bloch DA, McShane DJ, Fries JF, Cooper NS, Healey LA, Kaplan SR, Liang MH and Luthra HS: The American rheumatism association 1987 revised criteria for the classification of rheumatoid arthritis. Arthritis Rheum 31: 315-324, 1988.
14. Schmidt W, Schmidt H, Schicke B and Gromnica-Ihle E: Standard reference values for musculoskeletal ultrasonography. Ann Rheum Dis 63: 988-994, 2004.

15. Wakefield RJ, Balint PV, Szkudlarek M, Filippucci E, Backhaus M, D'Agostino MA, Sanchez EN, Iagnocco A, Schmidt WA, Bruyn GA, et al: Musculoskeletal ultrasound including definitions for ultrasonographic pathology. J Rheumatol 32: 2485-2487, 2005.

16. Gabriel SE: The epidemiology of rheumatoid arthritis. Rheum Dis Clin North Am 27: 269-281, 2001.

17. Cooles FA and Isaacs JD: Pathophysiology of rheumatoid arthritis. Curr Opin Rheumatol 23: 233-240, 2011.

18. Scott IC, Steer S, Lewis CM and Cope AP: Precipitating and perpetuating factors of rheumatoid arthritis immunopathology: Linking the triad of genetic predisposition, environmental risk factors and autoimmunity to disease pathogenesis. Best Pract Res Clin Rheumatol 25: 447-468, 2011.

19. Hutchinson D, Shepstone L, Moots R, Lear J and Lynch M: Heavy cigarette smoking is strongly associated with rheumatoid arthritis (RA), particularly in patients without a family history of RA. Ann Rheum Dis 60: 223-227, 2001.

20. Cutolo M and Straub RH: Stress as a risk factor in the pathogenesis of rheumatoid arthritis. Neuroimmunomodulation 13: 277-282, 2006.

21. Kahlenberg $\mathbf{J}$ and Fox D: Advances in the medical treatment of rheumatoid arthritis. Hand Clin 27: 11-20, 2011.

22. Nell V, Machold K, Eberl G, Stamm T, Uffmann M and Smolen J: Benefit of very early referral and very early therapy with disease-modifying anti-rheumatic drugs in patients with early rheumatoid arthritis. Rheumatology (Oxford) 43: 906-914, 2004.

23. Guillemin F, Billot L, Boini S, Gerard N, Ødegaard S and Kvien TK: Reproducibility and sensitivity to change of 5 methods for scoring hand radiographic damage in patients with rheumatoid arthritis. J Rheumatol 32: 778-786, 2005.

24. Hodgson RJ, O'Connor P and Moots R: MRI of rheumatoid arthritis image quantitation for the assessment of disease activity, progression and response to therapy. Rheumatology 47: 13-21, 2008.

25. Patil P and Dasgupta B: Role of diagnostic ultrasound in the assessment of musculoskeletal diseases. Ther Adv Musculoskelet Dis 4: 341-355, 2012.

26. Foltz V, Gandjbakhch F, Etchepare F, Rosenberg C, Tanguy ML, Rozenberg S, Bourgeois P and Fautrel B: Power doppler ultrasound, but not low-field magnetic resonance imaging, predicts relapse and radiographic disease progression in rheumatoid arthritis patients with low levels of disease activity. Arthritis Rheum 64: 67-76, 2012.

27. Amin MF, Ismail FM and El Shereef RR: The role of ultrasonography in early detection and monitoring of shoulder erosion and disease activity in rheumatoid arthritis patients; comparison with MRI examination. Acad Radiol 19: 693-700, 2012.

28. Szkudlarek M, Court-Payen M, Strandberg C, Klarlund M, Klausen T and Østergaard M: Power doppler ultrasonography for assessment of synovitis in the metacarpophalangeal joints of patients with rheumatoid arthritis: A comparison with dynamic magnetic resonance imaging. Arthritis Rheum 44: 2018-2023, 2001.

29. Rahmani M, Chegini H, Najafizadeh SR, Azimi M, Habibollahi P and Shakiba M: Detection of bone erosion in early rheumatoid arthritis: Ultrasonography and conventional radiography versus non-contrast magnetic resonance imaging. Clin Rheumatol 29: 883-891, 2010.

30. Orr JD, Sabesan V, Major N and Nunley J: Painful bone marrow edema syndrome of the foot and ankle. Foot Ankle Int 31: 949-953, 2010

31. McQueen FM and Ostendorf B: What is MRI bone oedema in rheumatoid arthritis and why does it matter? Arthritis Res Ther 8: 222,2006

32. Szopińska I, Kontny E, Maśliński W, Sobieszek M, Warczyńska A and Kwiatkowska B. Significance of bone marrow edema in pathogenesis of rheumatoid arthritis. Pol J Radiol 78: 57-63, 2013.

33. Schett $G$ and Gravallese E: Bone erosion in rheumatoid arthritis: mechanisms, diagnosis and treatment. Nat Rev Rheumatol 8: 656-664, 2012.

34. Jimenez-Boj E, Nöbauer-Huhmann I, Hanslik-Schnabel B, Dorotka R, Wanivenhaus AH, Kainberger F, Trattnig S, Axmann R, Tsuji W, Hermann S, et al: Bone erosion and bone marrow edema as defined by magnetic resonance imaging reflect true bone marrow inflammation in rheumatoid arthritis. Arthritis Rheum 56: 1118-1124, 2007.

35. Magnani M, Salizzoni E, Mulè R, Fusconi M, Meliconi R and Galletti S: Ultrasonography detection of early bone erosion in the metacarpophalangeal joints of patients with rheumatoid arthritis. Clin Exp Rheumatol 22: 743-748, 2004. 
36. Hoving JL, Buchbinder R, Hall S, Lawler G, Coombs P, McNealy S, Bird P and Connell D: A comparison of magnetic resonance imaging, sonography and radiography of the hand in patients with early rheumatoid arthritis. J Rheumatol 31: 663-675, 2004.

37. Alvarez-Nemegyei $J$ and Canoso JJ: Evidence-based soft tissue rheumatology: III: Trochanteric bursitis. J Clin Rheumatol 10 123-124, 2004.

38. Harcke HT, Grissom LE and Finkelstein MS: Evaluation of the musculoskeletal system with sonography. AJR Am J Roentgenol 150 1253-1261, 1988.

39. Kannegieter NJ: Chronic proliferative synovitis of the equine metacarpophalangeal joint. Vet Rec $127: 8-10,1990$.

40. Barile A, Sabatini M, Iannessi F, Di Cesare E, Splendiani A, Calvisi V and Masciocchi C: Pigmented villonodular synovitis (PVNS) of the knee joint: magnetic resonance imaging (MRI) using standard and dynamic paramagnetic contrast media. Report of 52 cases surgically and histologically controlled. Radiol Med 107: 356-366, 2004.
41. Kasukawa $R$, Takeda I, Iwadate $H$ and Kanno $T$ : Ultrasonographic evaluation of synovial effusion and synovial proliferation pattern in the knee joints of patients with rheumatoid arthritis. Mod Rheumatol 12: 64-68, 2002.

42. Ong CK, Lirk P, Tan CH and Seymour RA: An evidence-based update on nonsteroidal anti-inflammatory drugs. Clin Med Res 5: 19-34, 2007.

43. Gaffo A, Saag KG and Curtis JR: Treatment of rheumatoid arthritis. Am J Health Syst Pharm 63: 2451-2465, 2006.

44. Gorter SL, Bijlsma JW, Cutolo M, Gomez-Reino J, Kouloumas M, Smolen JS and Landewé R: Current evidence for the management of rheumatoid arthritis with glucocorticoids: A systematic literature review informing the EULAR recommendations for the management of rheumatoid arthritis. Ann Rheum Dis 69: 1010-1014, 2010.

45. Vlieland TP and Van den Ende CH: Nonpharmacological treatment of rheumatoid arthritis. Curr Opin Rheumatol 23: 259-264, 2011 